\section{P087 PROVIDERS' VIEWS ON BARRIERS AND FACILITATORS OF BACTERIAL STI TESTING AMONG GBMSM LIVING WITH OR AT RISK FOR HIV}

${ }^{1}$ Charlie Guiang ${ }^{*}{ }^{2}$ Ann Burchell, ${ }^{3}$ Jayoti Rana, ${ }^{4}$ Rita Shahin, ${ }^{5}$ Jason Brunetta, ${ }^{6}$ Leo Mitterni ${ }^{7}$ Darrell Tan, ${ }^{8}$ Jean Bacon, ${ }^{9}$ Mark Gilbert, ${ }^{10}$ Dionne Gesink, ${ }^{11}$ Ramandip Grewal, ${ }^{12}$ Carmen Logie, ${ }^{13}$ Anna Yeung, ${ }^{14}$ Ryan Lisk. ${ }^{1}$ St. Michael's, University of Toronto, Family and Community Medicine, Toronto, Canada; 'St. Michael's Hospital, Centre for Urban Health Solutions and Department of Family and Community Medicine, Toronto, Canada; ${ }^{3}$ St. Michael's Hospital, Li Ka Shing Knowledge Institute, Toronto, Canada; ${ }^{4}$ Toronto Public Health, Toronto, Canada; ${ }^{5}$ Maple Leaf Medical Clinic, Toronto, Canada; ${ }^{6}$ Hassle Free Clinic, Toronto, Canada: ' $5 t$ Michael's Hospital, 1. Division of Infectious Diseases, Toronto, Canada; ${ }^{8}$ OHTN, Toronto, Canada; ${ }^{9} B C$ Centre for Disease Control, Vancouver, Canada; ${ }^{10} \mathrm{U}$ of Toronto, Dalla Lana School of Public Health, Toronto, Canada; ${ }^{11}$ St Michael's Hospital, Toronto, Canada; ${ }^{12}$ University of Toronto, Factor-Inwentash Faculty of Social Work, Toronto, Canada; ${ }^{13}$ St. Michael's Hospital, Centre for Urban Health Solutions, Li Ka Shing Knowledge Institute, Toronto, Canada; ${ }^{14}$ AIDS Committee of Toronto, Toronto, Canada

\subsection{6/sextrans-2019-sti.282}

Background Bacterial STI testing is a necessary component of sexual health care for MSM living with and at risk for HIV. Guidelines recommend testing at least once a year or more often if at ongoing risk. As part of a larger mixed methods study with the overall goal to prioritize new STI testing interventions, our aim was to determine barriers and facilitators to offering bacterial STI testing to MSM according to healthcare providers in Toronto, Canada.

Methods In 06/2018-07/2018, we circulated invitations for an online, anonymous survey to an estimated 172 providers in Toronto. Providers were eligible if they provided care for $\geq 1$ MSM per week and were involved in the decision-making process in providing a STI test (e.g., taking sexual histories, ordering tests).

Results Of 93 respondents, 68\% worked in primary care, 32\% worked in public health/sexual health clinics, $70 \%$ were physicians and 30\% were nurses or other allied health professionals. Most (67\%) saw between 1-10 MSM clients per week. Among respondents working in primary care $(n=63)$, barriers to offering testing 'sometimes' or more often were: insufficient consultation time (64\%), difficulty introducing testing during unrelated consultations (52\%), forgetting to offer testing (46\%), patient reporting no sexual activity (30\%) and patient refusal (25\%). Among all respondents, preferred practice changes to improve testing were: express testing/fast-track testing services (89\%), provider alerts when patients are due for testing (87\%), selfcollected specimen sampling by patients (84\%), standing orders for tests (79\%), and nurse-led STI testing $(78 \%)$. Primary care providers were more in favour of provider alerts whereas providers at sexual health clinics favoured patient reminders.

Conclusion Among those whose practice incorporated sexual health care for MSM, providers were in favour of initiatives to simplify and expedite bacterial STI testing (including selfcollection of samples), prompts/reminders for testing, and expanding testing delivery to other healthcare professionals.

Disclosure No significant relationships.

\section{P088 \\ USING ELECTRONIC SCREENING AND FEEDBACK WITH ADOLESCENTS TO DECREASE SEXUAL HEALTH RISKS IN THE EMERGENCY DEPARTMENT}

${ }^{1}$ Taraneh Shafii, ${ }^{2}$ Siobhan Thomas-Smith* ${ }^{3}$ Miranda Bradford, ${ }^{4}$ Eileen Klein, ${ }^{1}$ Laura Richardson, ${ }^{1}$ Cari Mccarty, ${ }^{1}$ Taraneh Shafii. ' Seattle Children's Hospital/University of Washington School of Medicine, Adolescent Medicine/Pediatrics, Seattle, USA; ${ }^{2}$ Seattle Children's Hospital/University of Washington School of Medicine, Emergency/Pediatrics, Seattle, USA; ${ }^{3}$ Seattle Children's Hospital, Biostatistics/Pediatrics, Seattle, USA; ${ }^{4}$ Seattle Children's Hospital/University of Washington School of Medicine, Emergency Medicine/ Pediatrics, Seattle, USA

\subsection{6/sextrans-2019-sti.283}

Background Adolescents account for 1 in 4 sexually transmitted infections (STIs) diagnosed annually in the United States. Many adolescents seek care in emergency departments (ED) for acute medical problems which offers an opportunity to screen for risk behaviors including sexual health. As emergency medicine provider time is focused on the acute medical problem, using technology may be an acceptable and effective alternative to identify and decrease screening risky sexual behaviors.

Methods Adolescents aged 13-18 years participated in a randomized controlled trial of an electronic health behavior screening and feedback tool in a pediatric emergency department. All participants were surveyed about risky behaviors at the baseline ED visit and 3 months later, and only intervention arm participants received immediate individualized electronic feedback about their risk behaviors, normative comparisons and brief educational information, including on birth control and condoms. In this secondary analysis, we used Chi-squared analyses to compare differences in 3-month STI risk defined as sexually active and reporting inconsistent condom use.

Results A total of 296 adolescents including 55\% females enrolled in the study with $72 \%(n=212)$ completing the 3 month follow-up survey. At baseline 23\% (69/296) reported ever having sex and 67\% (46/69) reported using a condom at last sex. At follow-up 16\% (17/105) of the intervention group and $23 \%(25 / 107)$ of controls reported having sex in the last 3 months. Of those sexually active, 35\% (6/17) of intervention group compared to $56 \%(14 / 25)$ of control group reported not always using condoms in the last 3 months $(p=0.19)$. STI risk at 3 months was $6 \%(6 / 105)$ for intervention participants compared to $13 \%$ (14/107) for controls $(\mathrm{p}=0.07)$.

Conclusion Electronic sexual health screening in the emergency department increases opportunities to assess, identify, and intervene in risky sexual behaviors in adolescents and may contribute to a decrease in risky behaviors.

Disclosure No significant relationships.

\section{P089 EVALUATING ONLINE AND CLINIC-BASED STI SCREENING SERVICES: A CASE STUDY OF UMBRELLA SEXUAL HEALTH SERVICES, UK}

${ }^{1}$ Louise Jackson*, ${ }^{2}$ Nicola Thorley, ${ }^{1}$ Lorraine Munetsi, ${ }^{2}$ Jonathan Ross. ${ }^{1}$ University of Birmingham, Health Economics Unit, Birmingham, UK; ${ }^{2}$ University Hospital NHS Foundation Trust, Birmingham, UK

\subsection{6/sextrans-2019-sti.284}

Background Digital technologies are increasingly being adopted to increase STI screening uptake. From August 2015, the 